\title{
UCRL-TR-215093
}

LAW RENCE LIVERMORE N A T IO N A L LABORATORY
$\mathrm{CCl} 1$ and $\mathrm{CCl} 2$ Detector Simulations and Figure-of-Merit Study

D. Lange, H. Manini, D. Wright,

September 7, 2005 
This document was prepared as an account of work sponsored by an agency of the United States Government. Neither the United States Government nor the University of California nor any of their employees, makes any warranty, express or implied, or assumes any legal liability or responsibility for the accuracy, completeness, or usefulness of any information, apparatus, product, or process disclosed, or represents that its use would not infringe privately owned rights. Reference herein to any specific commercial product, process, or service by trade name, trademark, manufacturer, or otherwise, does not necessarily constitute or imply its endorsement, recommendation, or favoring by the United States Government or the University of California. The views and opinions of authors expressed herein do not necessarily state or reflect those of the United States Government or the University of California, and shall not be used for advertising or product endorsement purposes.

This work was performed under the auspices of the U.S. Department of Energy by University of California, Lawrence Livermore National Laboratory under Contract W-7405-Eng-48. 


\title{
CCI1 and CCI2 Detector Simulations and Figure-of-Merit Study
}

\author{
David Lange, Hugh Manini, and Douglas Wright \\ High Energy Physics Group \\ N Division \\ Lawrence Livermore National Laboratory
}

July 13, 2005

\begin{abstract}
We simulate the CCI1 and CCI2 detectors, using GEANT4, to study the figure of merit (FOM) for each detector. For both CCI1 and CCI2, we study how the FOM depends on strip pitch, $z$ resolution, and lever-cut distance. For CCI2, we study how the FOM depends on the separation distance between the two silicon detectors, and the separation distance between the two germanium detectors. We also simulate future large-scale detector systems and calculate their FOM.
\end{abstract}

\section{Detector simulations}

We do a full physics simulation, using GEANT4, of the CCI1 and CCI2 detectors. Only the detector crystals (silicon and germanium) are implemented in this version, with no other detector materials included. The CCI1 simulation geometry is shown in Figure 1. CCI1 consists of a silicon detector that is $64 \mathrm{~mm} \times 64 \mathrm{~mm}$ $\times 10 \mathrm{~mm}$ in size, and a germanium detector that is a circular disk which is $84 \mathrm{~mm}$ in diameter and $11 \mathrm{~mm}$ thick. The active portion of the germanium detector is a concentrically inscribed box which is $76 \mathrm{~mm} \times 76$ $\mathrm{mm} \times 11 \mathrm{~mm}$ in size. In Figure 1, the active portion of the germanium detector is the portion between the two dashed lines. The two detector crystals are separated by $5 \mathrm{~cm}$ center-to-center.

The CCI2 simulation geometry is shown in Figure 2. CCI2 consists of two silicon detectors that are each $64 \mathrm{~mm} \times 64 \mathrm{~mm} \times 10 \mathrm{~mm}$ in size, and two germanium detectors that are each circular disks which are $84 \mathrm{~mm}$ in diameter and $15 \mathrm{~mm}$ thick. The active portion of each germanium detector is a concentrically inscribed box which is $76 \mathrm{~mm} \times 76 \mathrm{~mm} \times 15 \mathrm{~mm}$ in size. In Figure 2, the active portion of each germanium detector is the portion between the two dashed lines. The separation distance between the silicon detector and the germanium detector is held fixed at $3 \mathrm{~cm}$.

Each GEANT4 simulation is run once for each specific geometry. Photons are generated at a specific energy and tracked through the detector volumes. The LLNL data tables (www-nds.iaea.org/epd197) for the compton and photoelectric effects are utilized in GEANT4 to model the photon interactions within the detector. We tally the interaction positions and kinematics for each interaction, and store this information for further analysis. 


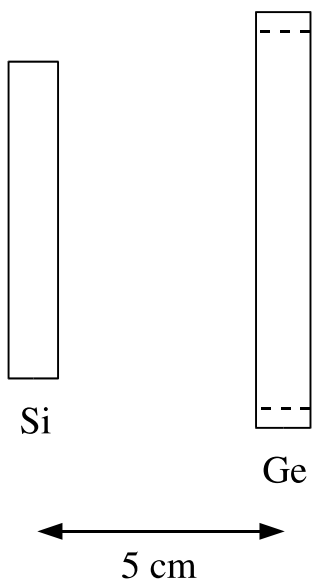

Figure 1: CCI1 detector geometry used in the simulation. The active portion of the germanium detector is the portion between the two dashed lines.

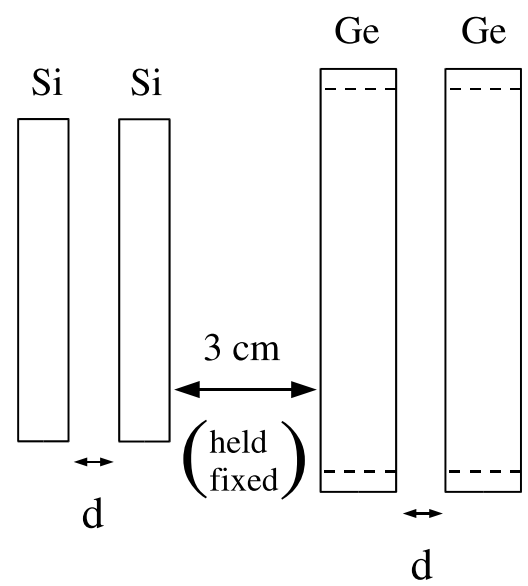

Figure 2: CCI2 detector geometry used in the simulation. The active portion of each germanium detector is the portion between the two dashed lines.

We then "digitize" the exact information and introduce detector resolution effects, so that the Monte Carlo output mimics the real data. The $x$ and $y$ coordinates are pixelized (converted into individual strip hits). When more than one true interaction occurs within a pixel, the interactions and deposited energies are combined. The strip energy distribution is determined by smearing individual energy deposits. We assume that each interaction is completely contained within a single strip. We do not follow the methods in real data for the determination of the $z$ (detected interaction depth within a detector) coordinate. Instead, the $z$ coordinate is determined by smearing the true $z$ location of the interaction using a Gaussian probability distribution. In each GEANT4 simulation, the source is positioned directly in front of the detector along the $z$ axis, 2 meters away from the detector, and 1.6 million signal events are generated for each input energy.

To study the variation in the FOM as we vary parameters, we use the same original GEANT4 input and only redo the digitization step when changing strip pitch or $z$ resolution. We vary one parameter, while holding the other fixed. The nominal value of the strip pitch is $2 \mathrm{~mm}$ and the nominal value of $z$ resolution is 
$1 \mathrm{~mm}$. For CCI2, the separation distance between the silicon detectors and the separation distance between the germanium detectors are each held fixed at the nominal value of $10 \mathrm{~mm}$ while we vary the strip pitch or the $z$ resolution. The nominal value of lever distance is 4 strip widths.

We also study the variation in the FOM of CCI2 as a function of the separation distance between the silicon detectors, and the separation distance between the germanium detectors.

\section{Figure of merit}

The figure of merit is $S / \sqrt{B}$ where $S$ and $B$ are calculated by integrating the plots of distance of closest approach (doca):

$$
\begin{aligned}
& S=\int_{0}^{\text {upper lim }} \text { doca }[\text { signal }](x) d x \\
& B=\int_{0}^{\text {upper lim }} \text { doca }[\text { background }](x) d x
\end{aligned}
$$

where the upper limit of integration defines the signal region boundary. The upper limit of integration is the radius of the signal region on the image plane. The distance of closest approach is the closest distance of an image ring to the true source position. The maximum figure of merit is found by varying the upper limit until the maximum value of $S / \sqrt{B}$ is achieved. Figure 3 shows a plot of figure of merit versus signal

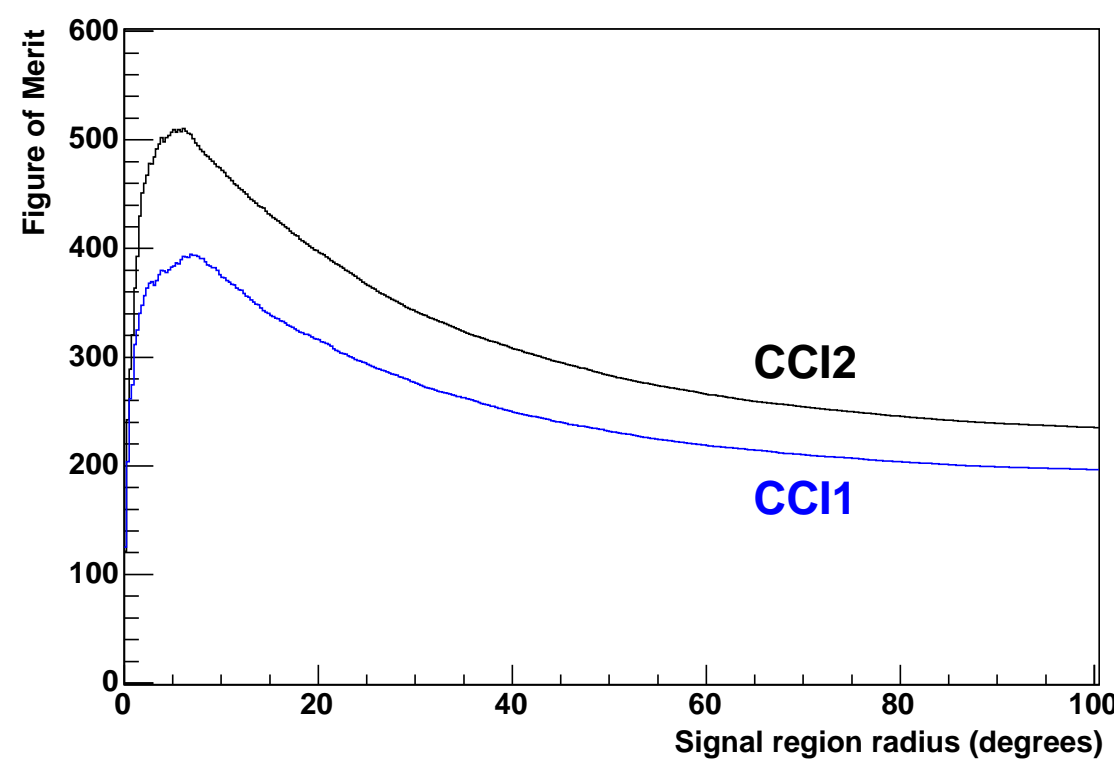

Figure 3: Figure of merit versus signal region radius for CCI1 and CCI2 with nominal parameters at 122 $\mathrm{keV}$, using the monoenergetic background model.

region radius for CCI1 and CCI 2 with nominal parameters at $122 \mathrm{keV}$, using the monoenergetic background model. The figure of merit curve for CCI1 reaches a maximum at a signal region radius of 7 degrees on the image plane. The CCI1 signal region with a radius of 7 degrees contains $66 \%$ of the total signal. The figure 
of merit curve for CCI2 reaches a maximum at a signal region radius of 6.25 degrees on the image plane. The CCI 2 signal region with a radius of 6.25 degrees contains $65 \%$ of the total signal.

\section{Background models}

Background photons are generated from $4 \pi$ surrounding the detector. We examine two background models. The first is a dirt-ball model calculation [1] using GAMGEN and MCNP from assumed isotopics for generic "dirt." Figure 4 shows a plot of the energy spectrum of the dirt-ball model as measured in the detector. The shelf in the dirt-ball spectrum at $90 \mathrm{keV}$ is produced by a $90 \mathrm{keV}$ cutoff imposed on the input photon spectrum. The portion of the spectrum below $90 \mathrm{keV}$ is produced by scatter-down from higher energies. Five source energies were chosen to sample the background at a range of different count levels. The red

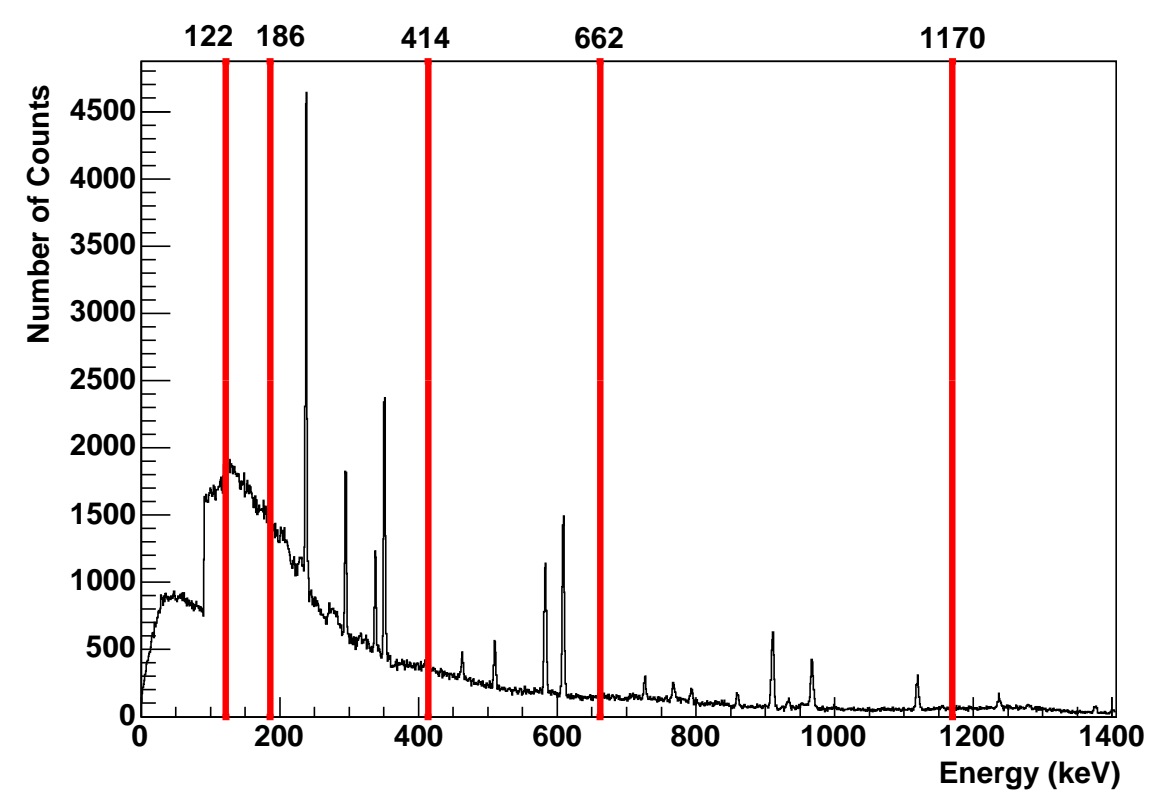

Figure 4: The energy spectrum of the dirt-ball background model. The red lines indicate the source energies at which the figure of merit is calculated.

lines indicate the source energies at which the figure of merit is calculated. We integrate the background within an energy window of $\pm 10 \mathrm{keV}$.

Since photons from the entire spectrum must be tracked in order to get all of the scatter-down contribution within any particular energy-cut window, it takes a significant amount of CPU time to accumulate sufficient statistics at high energies, where there is low incident flux. Low background statistics causes the background doca plots to be sparsely populated, which creates fluctuations in the values of maximum figure of merit.

To avoid this problem with the dirt-ball model, we also used a monoenergetic background model. In this model, photons with energies exactly equal to the source energy are generated. Since most of these generated background events will result in energy deposition within our energy window cut, it is easy to get good background statistics. The monoenergetic model neglects scatter-down from higher energies, however 
the FOM results using the monoenergetic model give similar results to those from the dirt-ball model, but without fluctuations caused by low background statistics.

In the dirt-ball model, a total of 40 million background photons are generated throughout the entire energy spectrum. In the monoenergetic model, 1.6 million background photons are generated at each source energy.

\section{CCI1 results}

Figure 5 shows plots of the CCI1 maximum FOM versus strip pitch, normalized to nominal, using the dirtball background model (left) and the monoenergetic background model (right). Decreasing the strip pitch produces better $x y$ resolution, which increases the FOM. The FOM curves do not exhibit any local maxima,
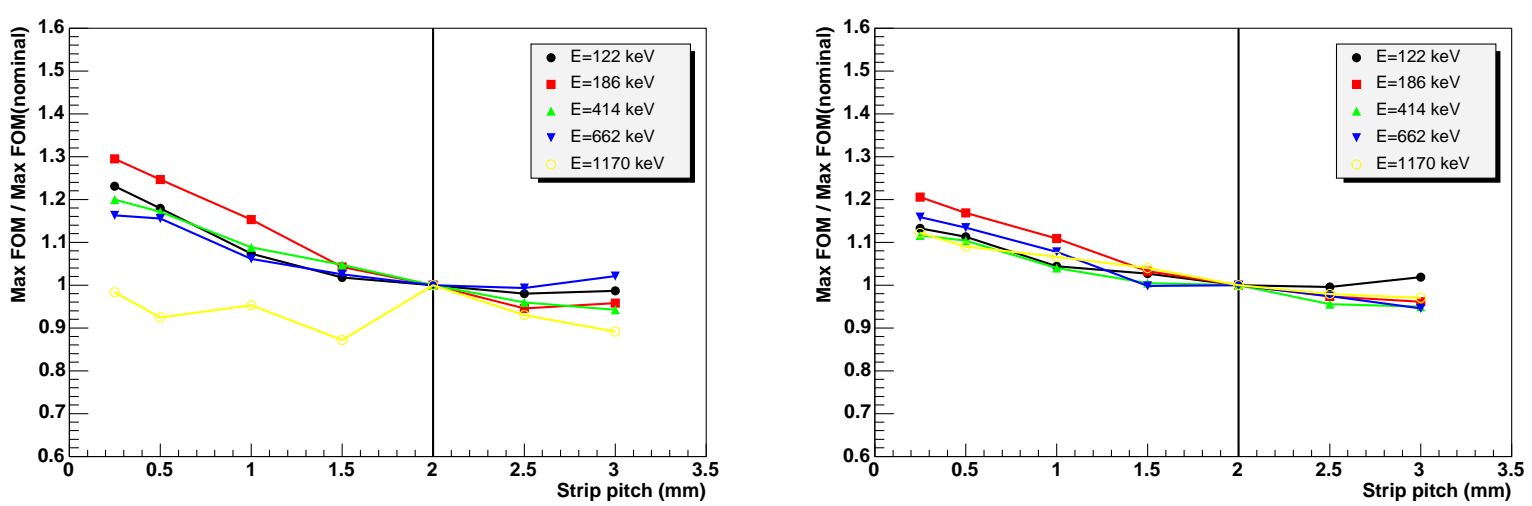

Figure 5: CCI1 maximum FOM versus strip pitch, normalized to nominal, using the dirt-ball background model (left) and the monoenergetic background model (right).

which suggests that the strip pitch should be made as small as possible. The background at $1170 \mathrm{keV}$ is low in the dirt-ball background model, causing the $1170 \mathrm{keV}$ background doca plots to be sparsely populated. This causes the large fluctuations in the $1170 \mathrm{keV}$ FOM plot using the dirt-ball model. Therefore, we report our remaining results using only the monoenergetic background model.

Figure 6 shows a plot of the CCI1 maximum FOM versus $z$ resolution, normalized to nominal, using the monoenergetic background model. Decreasing the position resolution in the $z$ direction increases the FOM. As expected, the $z$ resolution should be made as small as possible.

Figure 7 shows a plot of the CCI1 maximum FOM versus lever distance, normalized to nominal, using the monoenergetic background model. The lever distance is the distance between two interaction events in an individual scattering process in a detector crystal. The units of lever distance are strip widths. The lever cut eliminates all events which have a lever distance less than a specified value. Since the lever cut applies to both signal and background events, we did not find much variation in the FOM as a function of lever distance. 


\section{CCI2 results}

Figure 8 shows a plot of the CCI2 maximum FOM versus strip pitch, normalized to nominal, using the monoenergetic background model. Decreasing the strip pitch produces better $x y$ resolution, which increases the FOM for all energies except $122 \mathrm{keV}$. The FOM curves do not exhibit any local maxima, which suggests that the strip pitch should be made as small as possible.

Figure 9 shows a plot of the CCI2 maximum FOM versus $z$ resolution, normalized to nominal, using the monoenergetic background model. Decreasing the position resolution in the $z$ direction increases the FOM. As expected, the $z$ resolution should be made as small as possible.

Figure 10 shows a plot of the CCI2 maximum FOM versus lever distance, normalized to nominal, using the monoenergetic background model. The lever distance is the distance between two interaction events in an individual scattering process in a detector crystal. The units of lever distance are strip widths. The lever cut eliminates all events which have a lever distance less than a specified value. We find that the FOM increases as the lever distance increases.

Figure 11 shows a plot of the CCI2 maximum FOM versus separation distance using the monoenergetic background model. In Figure 11, the separation distance between the silicon detectors, and the separation distance between the germanium detectors, are varied together. Increasing the separation distance between the detectors produces fewer detected signal events, and thus decreases the FOM. This is because increasing the separation distance between the detectors decreases the detector solid angle for a photon to scatter a second time, which means that fewer signal image rings will be produced, and fewer events will contribute to the signal doca plot.

Figure 12 shows a plot of the CCI2 maximum FOM versus separation distance of the germanium detectors, using the monoenergetic background model. In Figure 12, the separation distance between the silicon detectors is held fixed at $5 \mathrm{~mm}$, and only the separation distance between the germanium detectors is varied. Increasing the separation distance between the germanium detectors decreases the FOM.

\section{Results for future large-scale detector systems}

The next detector to be developed is CCI3. The CCI3 detector is similar to CCI2, with two silicon detectors and two germanium detectors, but all four detectors are contained within one cryostat. The CCI2 system has both of its silicon detectors in one cryostat, and both germanium detectors in another cryostat. The silicon detectors and germanium detectors in CCI3 will have the same dimensions as those of CCI2. Placing all four detectors in one cryostat in $\mathrm{CCI} 3$ will reduce the amount of intervening material between the silicon and germanium detectors, which will improve detection performance. Also, since CCI3 will have all four detectors in one cryostat, the four detectors will be very close together. Decreasing the separation distance between the detectors increases the FOM. In our simulations of CCI3, the separation distance between each of the four detectors is $8 \mathrm{~mm}$.

The ultimate goal of this project is to build a large-scale Compton imaging (LSCI) detector system composed of a large array of CCI3 modules. We have done simulations of various LSCI systems and calculated the FOM for each system. Figure 13 shows the results of maximum FOM for various detector systems, plotted versus anticipated year of completion. The FOM for each detector system is calculated at $414 \mathrm{keV}$ using nominal parameters, and normalized to the maximum FOM of CCI2. The medium-scale Compton imaging (MCI) detector system is a $2 \times 2$ array of CCI3 modules. The LSCI $4 \times 4$ detector system is a $4 \times 4$ array of CCI3 modules, and the LSCI $8 \times 8$ detector system is an $8 \times 8$ array of CCI3 modules. 


\section{References}

[1] J. Gronberg, S. Johnson, D. Lange, D. Wright, Source and Background Gamma-ray Spectra, UCRL-TR-201636

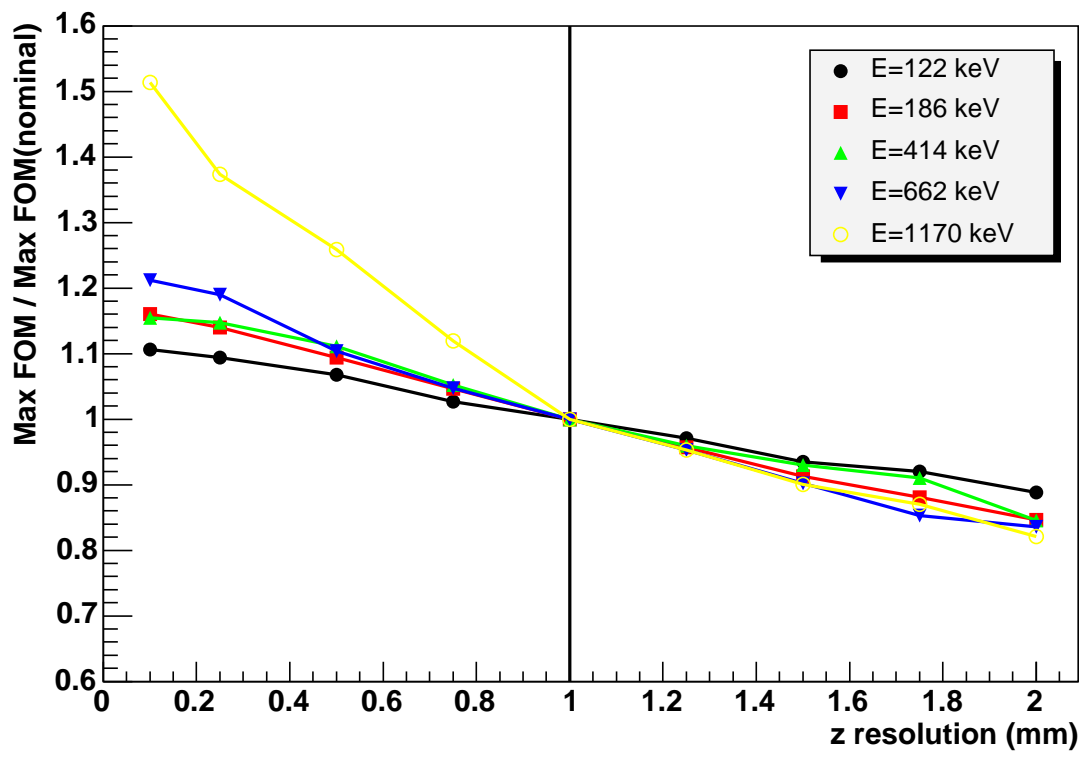

Figure 6: CCI1 maximum FOM versus $z$ resolution, normalized to nominal, using the monoenergetic background model. 


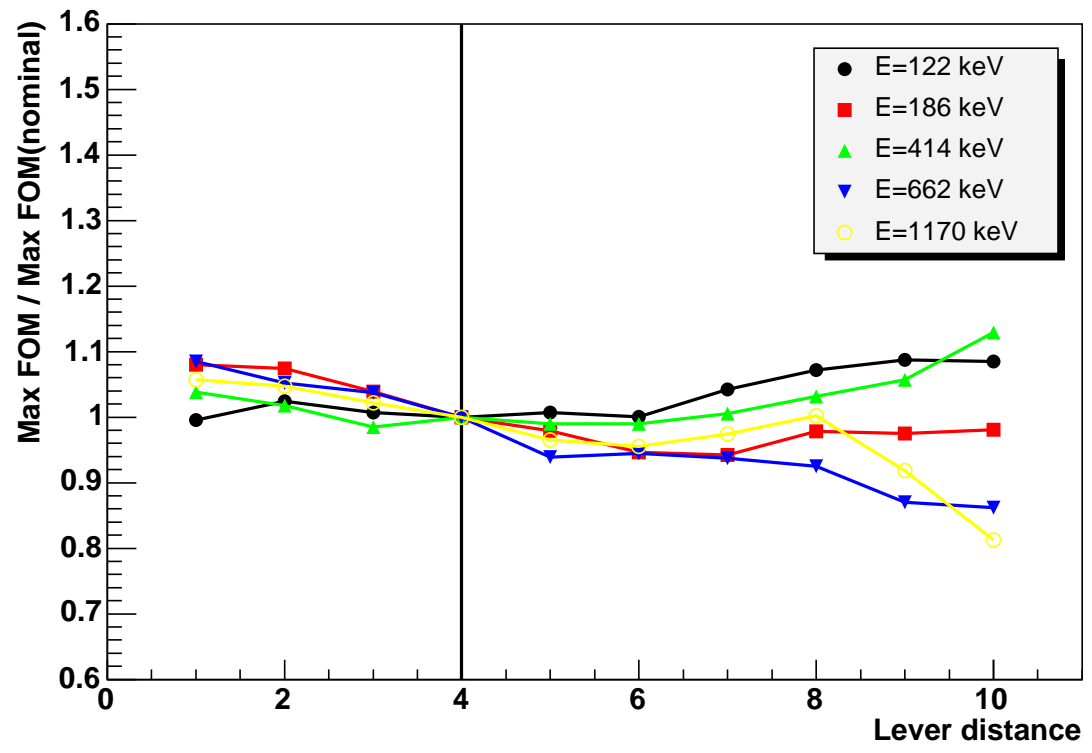

Figure 7: CCI1 maximum FOM versus lever distance, normalized to nominal, using the monoenergetic background model.

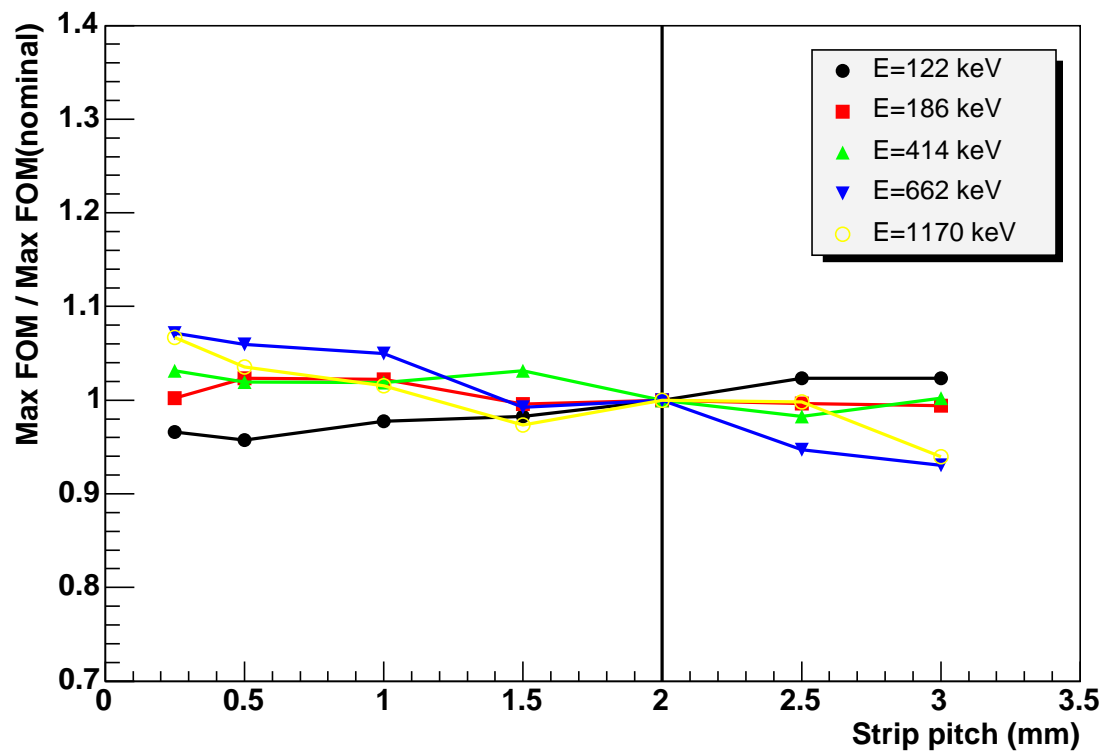

Figure 8: CCI2 maximum FOM versus strip pitch, normalized to nominal, using the monoenergetic background model. 


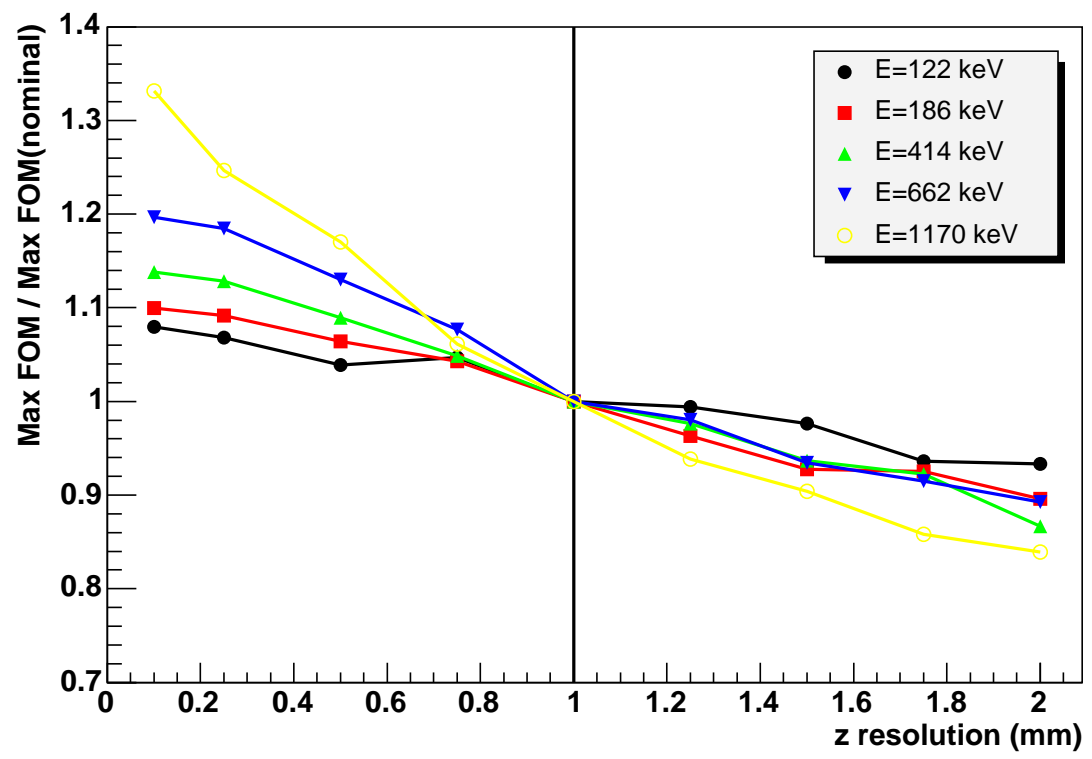

Figure 9: CCI2 maximum FOM versus $z$ resolution, normalized to nominal, using the monoenergetic background model.

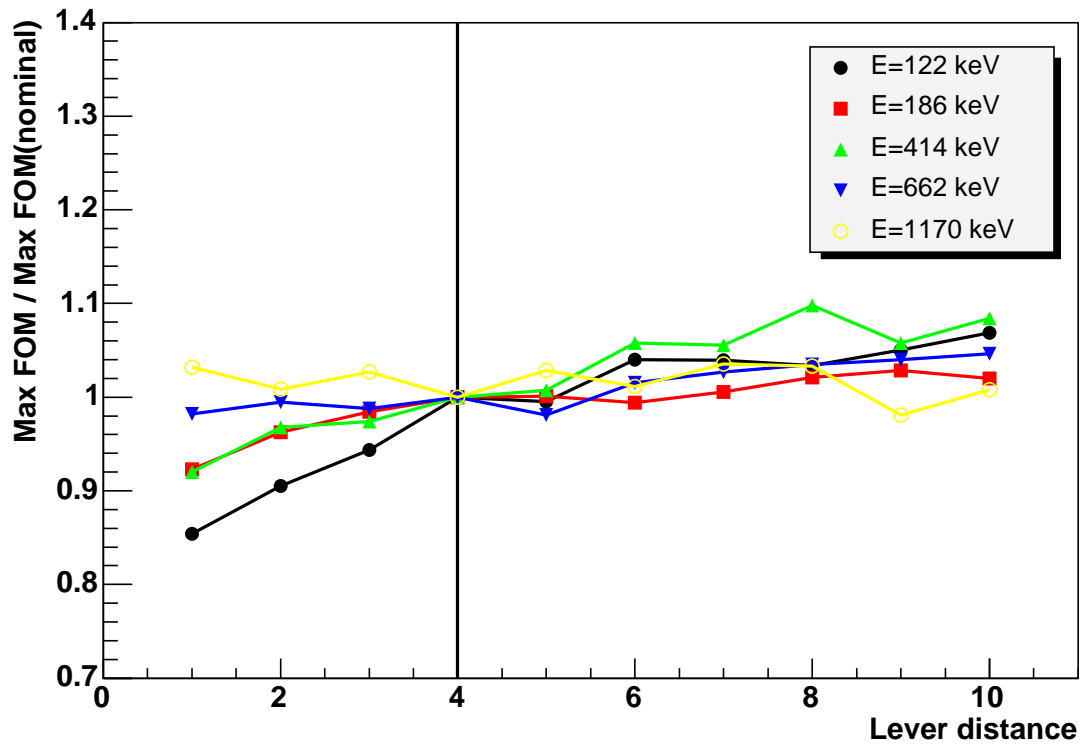

Figure 10: CCI2 maximum FOM versus lever distance, normalized to nominal, using the monoenergetic background model. 


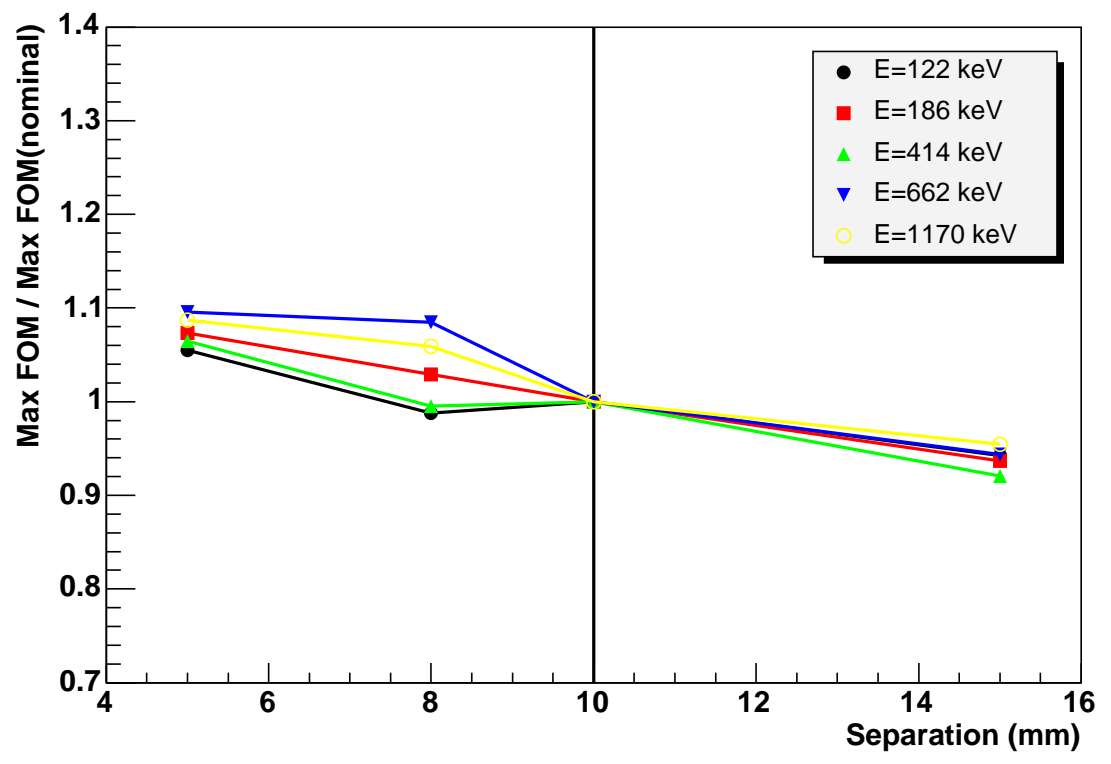

Figure 11: CCI2 maximum FOM versus separation distance, normalized to nominal, using the monoenergetic background model. The separation distance between the silicon detectors, and the separation distance between the germanium detectors, are varied together.

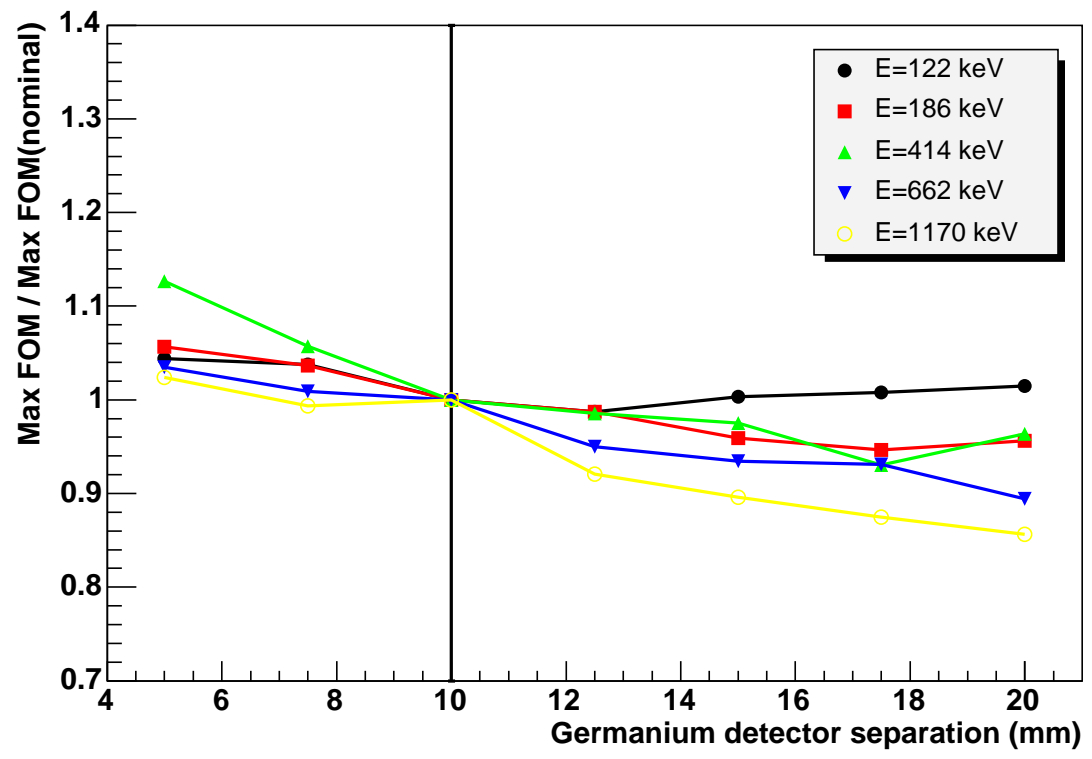

Figure 12: CCI2 maximum FOM versus separation distance of the germanium detectors, normalized to nominal, using the monoenergetic background model. The separation distance between the silicon detectors is held fixed at $5 \mathrm{~mm}$, and only the separation distance between the germanium detectors is varied. 


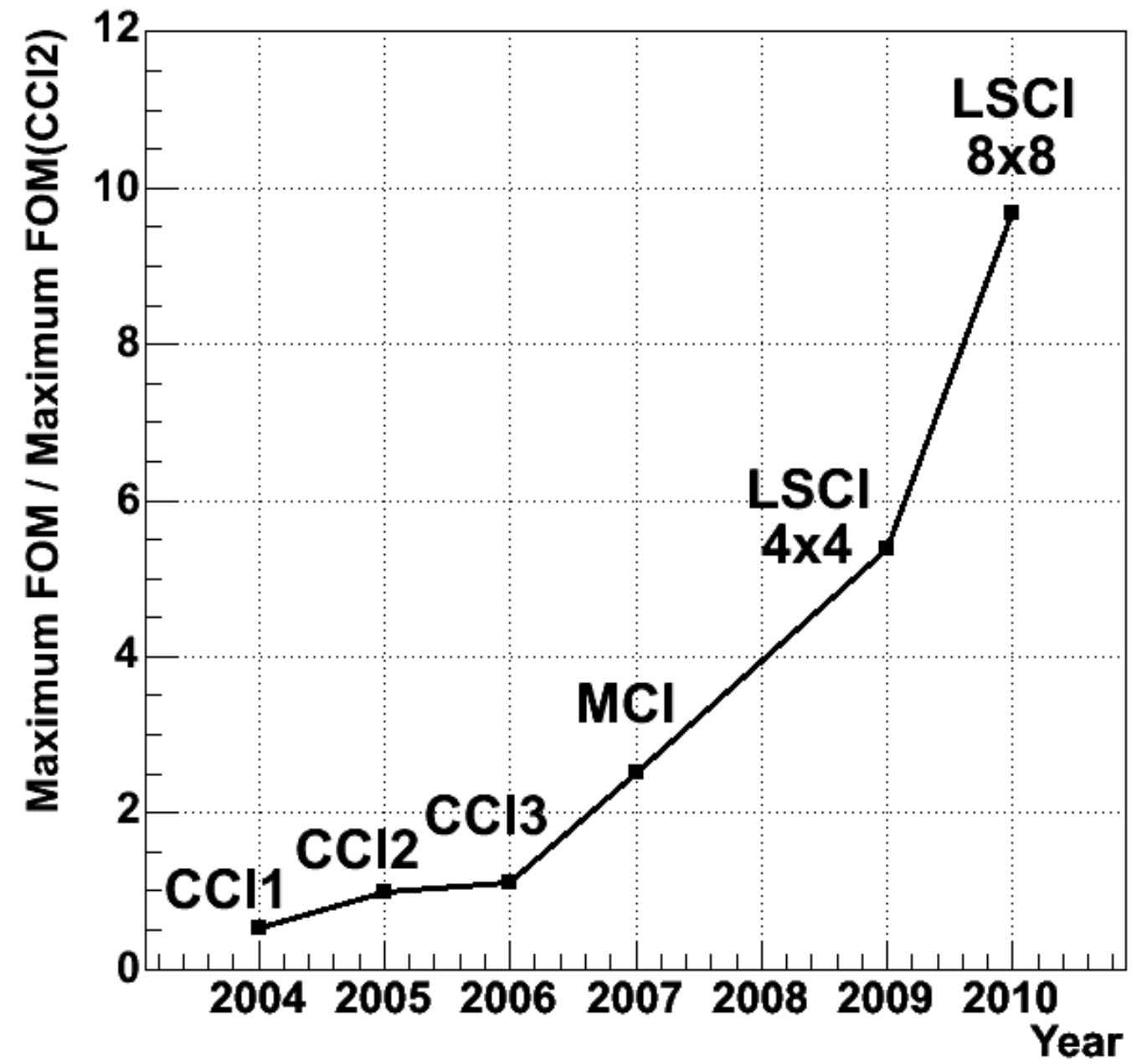

Figure 13: Maximum FOM for various detector systems, plotted versus anticipated year of completion. The maximum FOM for each detector system is calculated at $414 \mathrm{keV}$ using nominal parameters, and normalized to the maximum FOM of CCI2. The medium-scale Compton imaging (MCI) detector system is a $2 \times 2$ array of CCI3 modules. The large-scale Compton imaging (LSCI) $4 \times 4$ detector system is a $4 \times 4$ array of CCI3 modules, and the LSCI $8 \times 8$ detector system is an $8 \times 8$ array of $\mathrm{CCI} 3$ modules. 